\title{
Increasing of Precision Technology of Glass Sorting Based on Very Fast Reconfigurable Image Processing
}

David Krcmarik, Michal Petru, Ivan Masin

Technical University of Liberec, Studentská 2, 461 17, Liberec 1, Czech Republic. E-mail: michal.petru@tul.cz, david.krcmarik@tul.cz, ivan.masin@instituti.cz

The paper deals with a method and technology for increasing of precision sorting of transparent materials - glass. The recycled glass is a valuable material which is necessary as an ingredient in the process of glass manufacturing. We propose a very fast industrial device based on FPGA (field-programmable gate array) image processing along with a novel algorithm for a robust real-time treatment of acquired data. The system consists from a line-scan camera with fast CameraLink interface, FPGA processing, battery of valves propelled via pneumatics and nozzle block. Very important part of the system is a background configuration software enables precise assessment of required limits as inputs to FPGA processing. The overall performance is discussed from the perspective of laboratory and industrial tests. The proposed FPGA image processing can be utilized for further future enhancements in the field of precise sorting of glass with stuck labels on it. The results give very good performance even in the industrial environment with a lot of dust and dirt and hence the glass does not need to be extensively cleaned.

Keywords: Glass cullet; sorting; optical processing; FPGA; color extraction; pneumatics.

\section{Introduction}

Glass is very good recyclable like in comparison with polymeric materials [25]. Up-to-date goal of glass industry towards higher level of glass products recycling is to use as much glass cullet as possible [1]. The use of cullet avoids $\mathrm{CO} 2$ emissions, since cullet requires less energy to melt, and replaces carbonated raw materials. Although significant effort has been made by the upstream European container glass industry, especially through cullet recycling whose rate has increased from 43 $\%$ in 1990 to $73 \%$ in 2015 [2], further improvements are required to reduce its global environmental footprint, enhancing not only cullet use but also improving its quality [3]. Glass cullet can also be used in the construction industry for production ceramic bricks, tiles and their glazing, glass-ceramics, foam glass-ceramics, porcelain [4] or as a light composite material for transport applications resp. alternative for carbon composites [26,27]. An essential condition for recycling glass is efficient, fast and precise sorting of glass cullet. Glass sorting and separating methods and machines have accordingly been studied and discussed for more than fifty years [5-9]. General glass sorting systems (Fig. 1) typically consist of the following five sub-systems:

- feeding sub-system ensuring the transfer of the mixture of particles into the inspection zone

- inspection sub-system ensuring the measurement or detection of physical properties of particles

- evaluation sub-system ensuring comparison of actual and required values and transmit signals for rejecting or accepting of measured particle

- sorting sub-systems ensuring by means of actuators physical action on the particles

- collecting sub-system ensuring the position of the sorted particles.

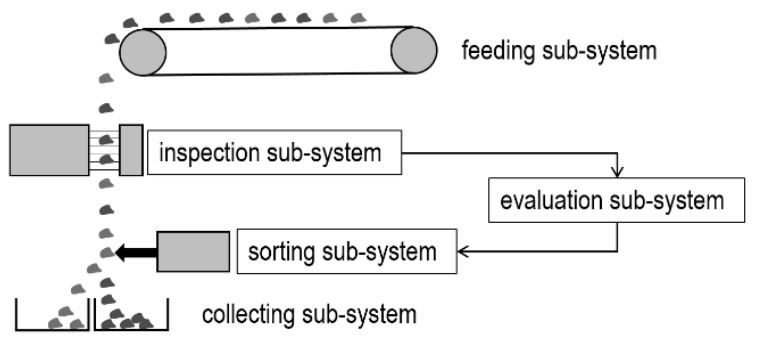

Fig. 1 General glass cullet sorting system

Glass sorting processes are based on the evaluation of physical characteristics of sorted material or particle. For this evaluation mechanical, optical, chemical or electrical effects are usually exploited. Historically and currently used cullet sorting methods include:

- $\quad$-sorting as a function of density [10]

- $\quad$ sorting by differential thermal characteristic [11]

- $\quad$ froth flotation [12]

- $\quad$ electrostatic separation [12]

- $\quad$ electro-optical sorters that recognize the color of cullet based on their opacity [5]

- $\quad$ sorting by transmission of visible light at certain wavelengths to distinguish between clear, brown and green glass [8, 13, 14, 15, 16]

- $\quad$ sorting using camera with high power multispectral LED light source [17]

- -spectrophotometric techniques using laser beam light $[18,19,20]$

- $\quad$-spectroscopy using UV absorption [21, 22]

- $\quad$ sorting using X-ray fluorescence spectroscopy [23]

- $\quad$ optical sorting usingan array of optical vortices [24] 
In terms of the steadily increasing volume of recycled glass and increasing demands for productivity and quality of sorting process, image processing methods and spectroscopic methods have the greatest potential from the above listed methods. The subject of our research was therefore sorting technology based on image processing. Considering that the increase in sorting productivity (efficiency) is influenced on the one hand by the image processing speed factor (equivalent of production machine cycle time) and on the other side by the factor of sorting machine reconfiguration time (equivalent of production machine changeover time), we are discussing the possibilities of improving the sorting machines in terms of these factors.

\section{Materials and methods}

This paper deals particularly with final stage of cullet sorting process - glass sorting machine. The sorting machine consists of two parts hardware and software. First we will deal with hardware.

\subsection{Hardware}

We have used a traditional configuration of using compressed air for blowing out desired/undesired pieces. The input to the glass sorting machine is glass cullet with CSP without any organic material. The principal scheme is depicted in Fig. 2. The vibration feeder needs to have a gratual circucalar transition into chute for cullets. If this is not met the cullet are jumping on the chute instead of sliding. Since the cullet is very abrasive we have found that optimal material is glass. Tests with stainless steel showed its impropriety. At the end of glass chute just before its end there is a high intensity LED light ( 3 segment parallel beam Corona II) with a diffuse piece of plastic or glass in order to have uniformly distributed the light along the whole width of the chute. This end of glass chute serves as an area of detection which is sensed by a line camera (Basler spL 4096-39kc). Few centimeters below is a set of nozzles (in line holes with $1 \mathrm{~mm}$ diameter apart from $6 \mathrm{~mm}$ ) which are propelled by individual valves (BN 16 watts, 80 psi, open delay $3,88 \mathrm{~ms}$, close delay $1,84 \mathrm{~ms}$ ). Each valve has an equal lenght hose connecting it to a proper nozzle. The valves are initialized by printed circuit board which is controlled by a supervisor FPGA based logic. The line camera, area of detection and the light are all in one line, hence the camera is looking directly into the light. This approach of sorting is usable only for transparent materials. However if the light is placed on the same side from area of detection as the camera, the machine could be used also for non-transparent materials.

A few centimeters below is the decision edge which has to be properly adjusted. The overall view on the machine is visible in Fig. 3.

\subsection{FPGA}

Quite unique is the software which is the heart of the machine. It consists of two parts - FPGA real-time control and off-line configuration GUI. The block diagram of the FPGA software is depicted in Fig. 4. The used FPGA is within a System on the chip (SOC) using a Zynq technology - MicroZed Zynq 7020.

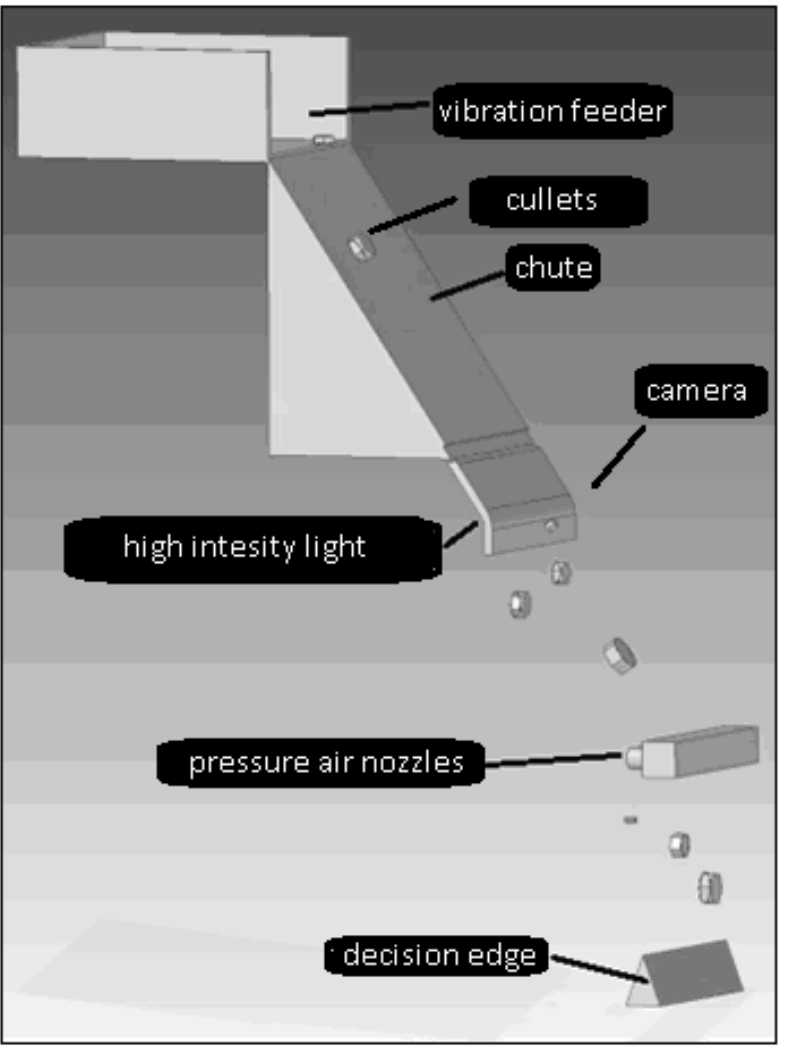

Fig. 2 Principal scheme of the main part of machine sorting

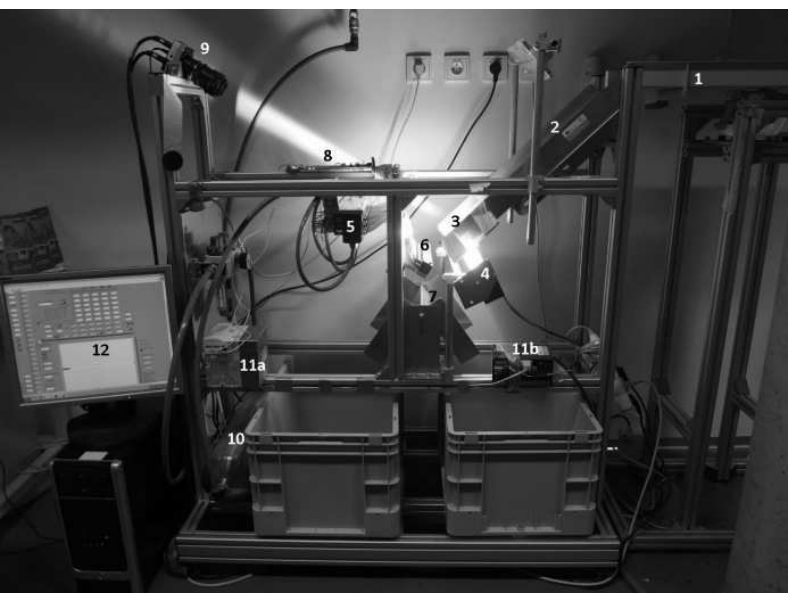

Fig. 3 Machine scheme: 1 -vibration feeder, 2 -glass chute, 3 - area of detection, 4 - lights, 5 -nozzle block, 6 -valve block, 7 -decision edge, 8 -valve control unit, 9 - line camera with camera link interface, 10 - pressured air buffer, 11 - power, 12 - PC with FPGA board

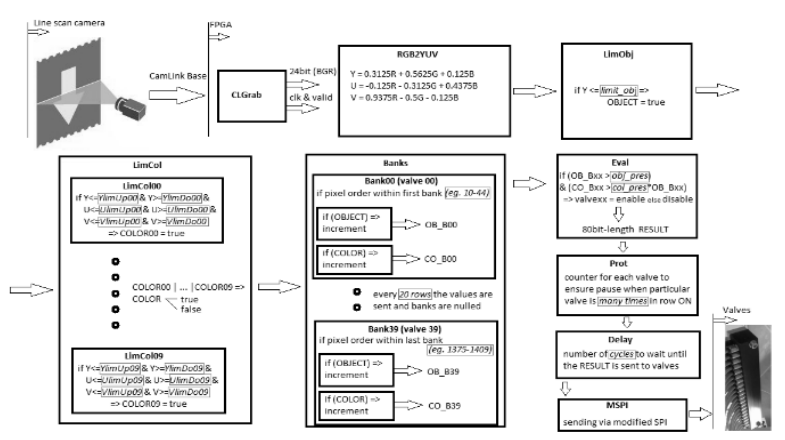

Fig. 4 Block diagram of FPGA data processing (user inputs are in green boxes) 


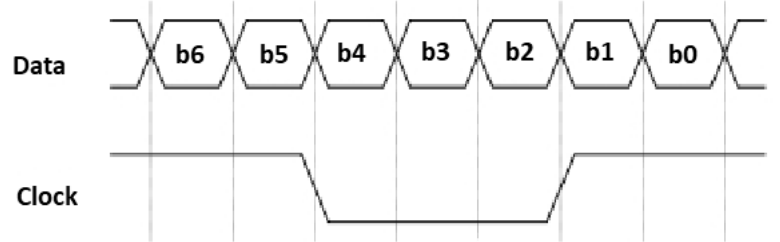

Fig. 5 Serialization of data in CameraLink protocol

The line scan camera provides continuously 2048 color pixels (camera uses Bayer encoding). Each pixel consists from red, green and blue component hence resulting in $24 \mathrm{bits} /$ pixel. The exposure time of line scan camera was chosen as small as possible $-80 \mu \mathrm{s}$. The data from all 2048 color pixels are sent every $82 \mu \mathrm{s}$. The protocol used to send data from camera to FPGA is CameraLink Base (4 differential parallel data lines) 3tap, each color coded to 8 bits and clock speed $40 \mathrm{MHz}$. The overall data throughput is quite high $699 \mathrm{MHz}$. The CameraLink protocol enables such a high throughput thanks to using 4 parallel data lines and embedded serialization of data - in other words upon one clock tick on each data line come 7 bits of data - Fig. 5. Data coming to FPGA are processed in CLGrab block which deserializes data (clock speed of CameraLink is $40 \mathrm{MHz}$ ). As a result CLGrab block gives for each pixel RGB components and also provides if currently read data are valid and internal pixel clock. Data are converted from RGB space into YUV because YUV space better suits needs for sorting. The conversion is in RGB2YUV block. The coefficients for conversion ( $\mathrm{Y}=$ $0.3125 \mathrm{R}+0.5625 \mathrm{G}+0.125 \mathrm{~B}, \mathrm{U}=-0.125 \mathrm{R}-0.3125 \mathrm{G}+$ $0.4375 \mathrm{~B}, \mathrm{~V}=0.9375 \mathrm{R}-0.5 \mathrm{G}-0.125 \mathrm{~B}$ ) are chosen in such a way to be fixed point values with 4 decimal places. This is because of FPGA processing. Next blocks operate with user inputs which are provided from off-line user interface. First we have to clarify the algorithm strategy used. The obtained image from camera when visualized can be typically as shown in Fig. 6. In fact camera gives at certain time only one row (e.g. $\ln 01, \ln 02$ ). So the shown image is a collection of several lines (namely lines within rows A to K) over some time. The lines can even overlap depending on the speed of the falling glass (typical values are around $2.2 \mathrm{~m} / \mathrm{s}$ ) and on the shutter of the line scan camera. In either case the functionality does not change. The cullet moves from up to down. We group always a certain number of consecutive lines into a single row for example lines $\ln 01$ up to $\ln 20$ are grouped into row $\mathrm{A}$, then line count starts again and other 20 lines are grouped into row B. Similarly we group the pixels into columns. So for example the camera is put into the sorting machine in such a way that the area of detection starts at pixel 10 and ends with pixel 569. First 35 pixels (e.g. px10 to px 44) are then grouped into column 1 then next 35 pixels are grouped into column 2 etc. It is necessary to point out that our rows and columns are only virtual. Rows are guided by the time and columns are guided by the position of the pixels within the sensing chip. The pixel count which forms a single column was chosen in such a way that a certain nozzle propelled with a particular valve is blowing air into this pixel area. The line count which form $\mathrm{s}$ a single rows was chosen accordingly to pixel count in such a way that a particular cell defined by column and row forms approximately a square although this is not necessary for correct operation. The pixel count, line count and start pixel of area of interest are all user configurable.

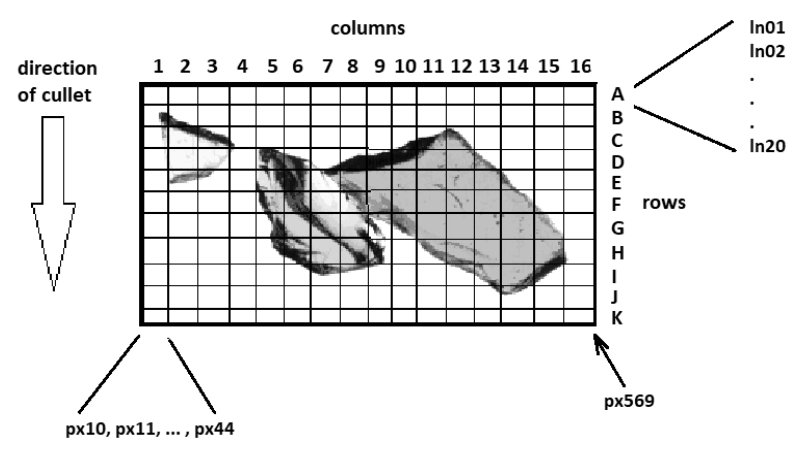

Fig. 6 Part of typical image from camera

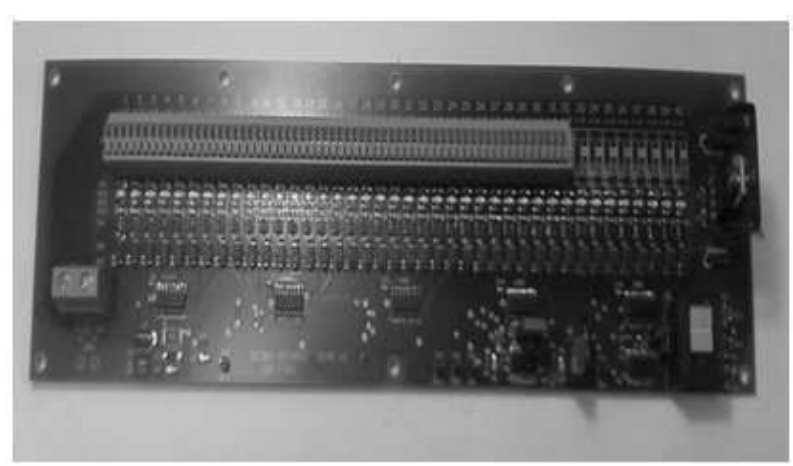

Fig. 7 Controlling PCB for valves

So each pixel belongs to a certain cell. Each cell (bank) is treated separately. The user has to choose several other parameters within GUI. Parameter called limit_obj (see Fig. 4) defines a maximal Y component of a pixel which describes a potential pixel of an object (glass or CSP) - it is usually around 245 (span of Y is from 0 to 255 - 255 means white color which means there is no obstacle between light source and camera). The user has to define also up to 10 sets of senary numbers - upper limit of $Y$, lower limit of $Y$, upper limit of $U$, lower limit of $U$, upper limit of $\mathrm{V}$ and lower limit of $\mathrm{V}$. If all particular pixel components fall within such limits (or another 9 sets of limits), the pixel is said to fulfill the condition for the sorted color. Hence every pixel is tested for an „object ${ }^{\text {“ }}$ and for a „,color“. If ,object“ (OBJECT) is met the particular bank to which pixel belongs is incremented and similarly if ,color" (COLOR) is met the particular bank is also incremented. After each 20 lines forming one virtual row we evaluate the counters for OBJECT and COLOR for each bank (cell) and if the conditions (1) and (2) are both met a particular valve is labeled as Enabled.

$$
\begin{gathered}
\text { OB_Bxx }>\text { obj_pres } \\
\text { CO_Bxx }>\text { col_pres } * \text { OB_Bxx }
\end{gathered}
$$

Otherwise a particular valve is labeled as Disabled. In equations (1) and (2) OB stands for OBJECT, CO stands for COLOR, Bxx marks a particular bank (eg. B20 is Bank20) and two left parameters obj_pres and col_pres 
are user defined. Parameter obj_pres describes the percentage of cell which has to be filled with any part of falling object (e.g. for pixel count 35 and line count 20 we have maximum of 700 potential pixels, if obj_pres is set to 350 it says that if at least $50 \%$ of pixels in a bank belong to potential object we have to consider that there is a object of interest). Parameter col_pres is a fixed point decimal number with 5 decimal points describing what percentage of object pixels within a bank has to meet the any of the 10 color specification set for a particular color to sort in order to really enable the valve. So the core of the real-time evaluation is within the blocks LimObj, LimCol, Banks and Eval (see Fig. 4). Valves have specification which defines the maximum allowed time when enabled. This time for our valves is around $0,5 \mathrm{~s}$. This is due to fact that there is a high current flowing into the valves when on and they need to cool down. Normally the valves are on only a small amount a time. But just as a protection a special counter which control the amount of continuous operating time for each valve is implemented. If such a counter is exceeded the particular valve is forced for a certain time to be off. Next block is a delay block. As shown in Fig. 2 there is a certain distance (in our case cca $10 \mathrm{~cm}$ ) between the area of detection and actual air pressure nozzles. So the 80bit-length RESULT is stored for a certain amount of cycles (cycles is a virtual row time duration) and after that is fed into MSPI (proprietary modified SPI) interface to the printed circuit board (PCB) which controls each valve - Fig. 7 .

\subsection{Graphical user interface}

The off-line graphical user interface is very important since there are many parameters which have to be configured into FPGA. The interface greatly simplifies the process. It is written in C\# and interfaces the FPGA via ARM processor. The communication link between the configuration PC and MicroZed is via Ethernet so it is even possible to configure the FPGA remotely. In ARM processor there is set of functions which call drivers directly communicating with appropriate parts of FPGA. More details about the software is behind the scope of this article. User interface provides several options - image grabbing, assesment of user parameters (parameter in green from Fig. 4) based on the color which is wanted to be sorted, serial interface for sending selected parameters to FPGA and testing of individual valves. The image grabbing option is implemented as a circular buffer of obtained lines from line scan camera which are saved into DDR3 which is part of MicroZed. User has several options of start/stop grabbing. Either using a GUI buttons, or use buttons on MicroZed board or the grabbing can be started by an internal event. The last option is mostly used. The user simply takes fistful of material to be sorted and cast it into the sorting machine. The program automatically start grabbing the image after first detection of object. After that user can download the image and start to process it. Found parameters are saved under a certain profile (e.g. profile Green glass, Brown glass, CSP, ...) and using a built in serial interface send to the FPGA. FPGA stores the data into an SD card so after restart is immediately starts to do its jobs according to the last configuration made. This serial communication is easily feasible since CameraLink standart has two differential lines for sending and receiving data (SerTC, SerTFG). Testing is needed because the valves from time to time stop working properly. Hence user can active arbitrary valve and check for proper function.

\section{Results and discussion}

One of the keys which are crucial for proper function is to choose before mentioned parameters appropriately. Along with the unique described algorithm this is a very important thing. In Fig. 8 there is a GUI with obtained image from the line scan camera.

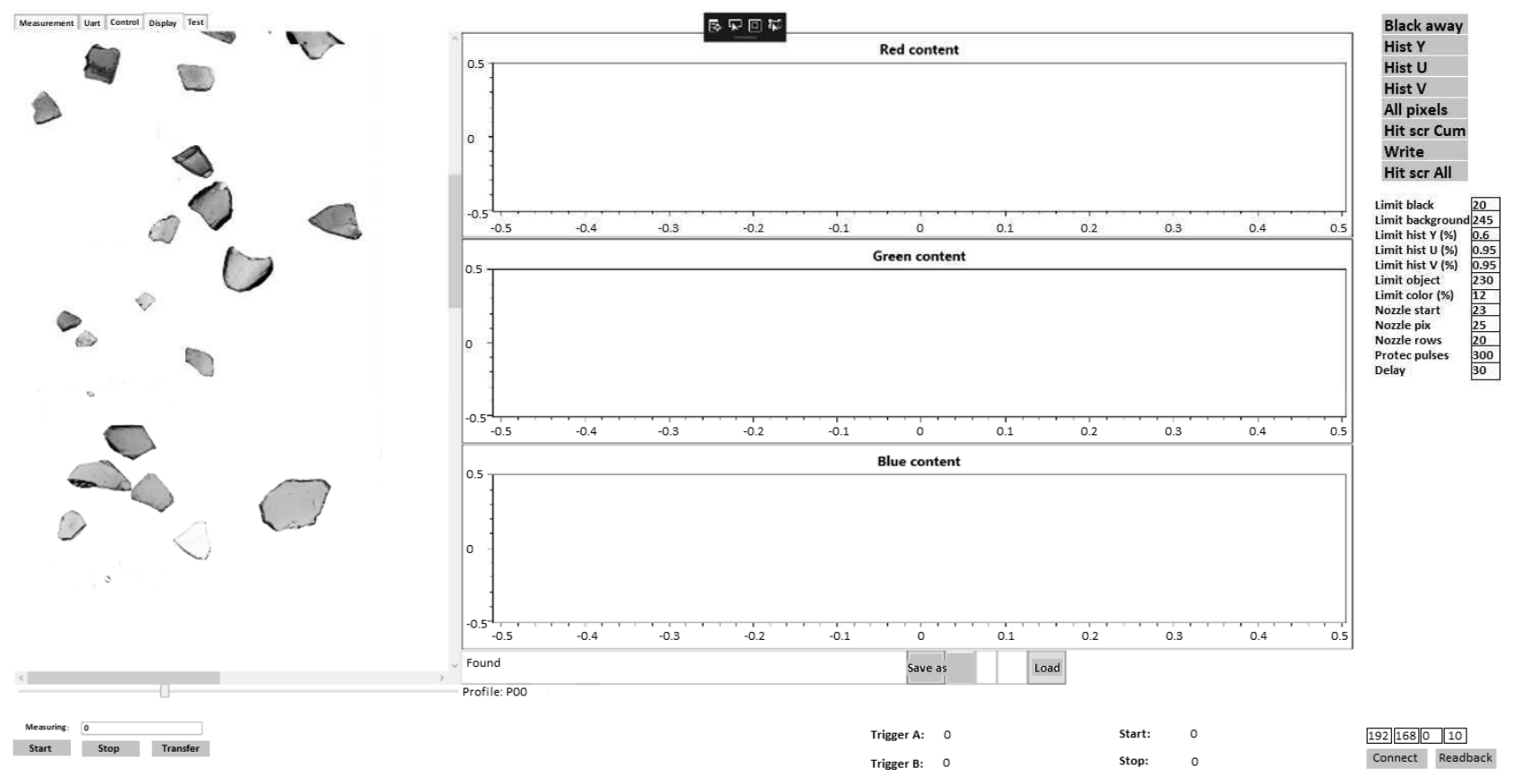

Fig. 8 Initial GUI 


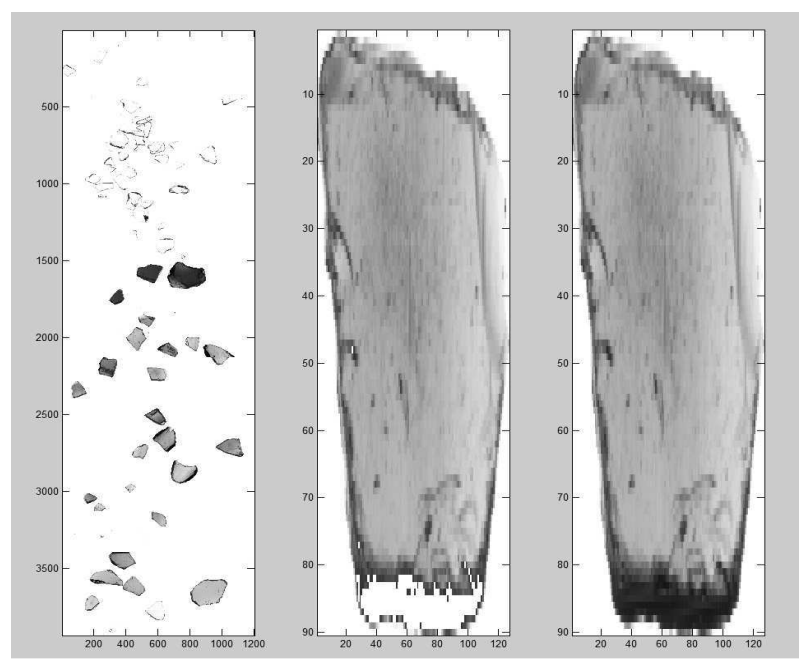

Fig. 9 Ignoring black content $(Y<20)$
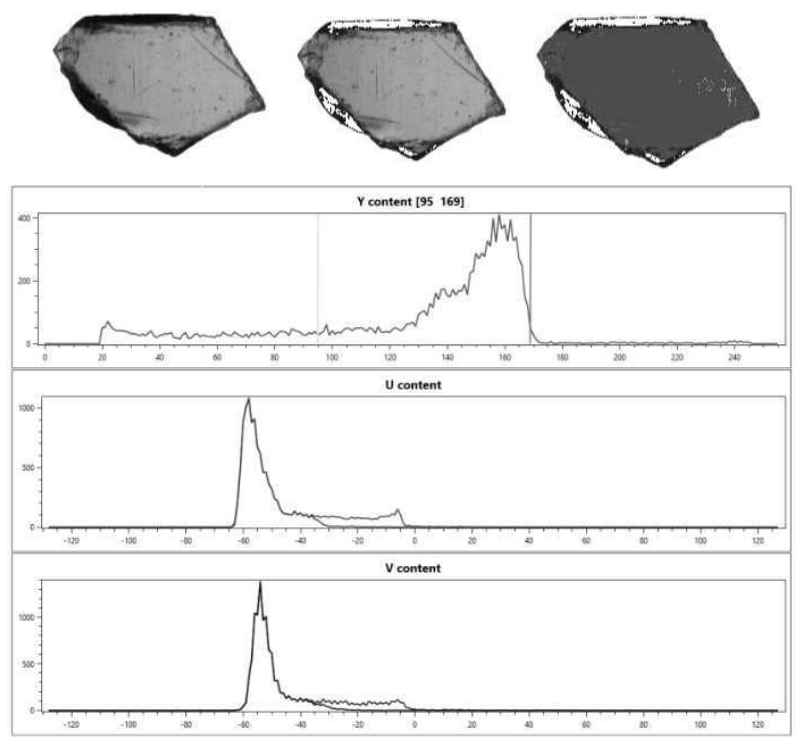

Fig. 10 Found Y limits with required portion of histogram $80 \%$, in $U$ and $V$ histograms are visible two graphs as a result of pixel chosen with the condition $95<=Y<=169$ (such pixels shown in red above)

Let's consider a case that a user wants to create a new profile which would blow out all green glass and rest will stay. First of all he has to define a value for parameter limit_obj (see Fig. 4). This gives the information which pixels are considered as object pixels and which are only background (background can be formed by a dust which could by deposited for some time on the chute until it is brushed away by cullet). First he has to select with a click a particular piece of glass of interest. Then using the buttons in the upper right corner he starts to process the piece of glass. He defines the limit of black which he wants to ignore (typically $\mathrm{Y}<20$ ). The glass on the edges has nearly always artifacts due to the refraction of light which gives his edges very dark color. We want to avoid such color not to come into our process. The result is on Fig. 9. Next he use three buttons "Histogram Y", "Histogram U" and "Histogram V" which computes appropriate quantities. Using text boxes defining wanted portion of selected histograms, the user can set limits on Y, U and V which satisfactorily satisfie his need. It is advisable to use such portions of histograms so that the resulting Y, U, V limits are as much as possible restrictive. If they are too wide the pixels which satisfy the conditions can occur also within glass pieces which are not wanted to sort Fig. 10. When chosen $Y$ limits, the $U$ histogram is slightly changes according to the restrictions of $\mathrm{Y}$ and similarly the same applies for $\mathrm{V}$ limits with respect to $\mathrm{U}$ (Fig. 11). Also the amount of pixels which satisfy the conditions for $\mathrm{Y}$ and $\mathrm{U}$ is less then only for $\mathrm{Y}$ condion (similarly for conditions $\mathrm{Y}, \mathrm{U}$ and $\mathrm{V}$ with restpect to $\mathrm{Y}$ and $\mathrm{U}$ ) - see red dots in a piece of glass in Fig. 11.

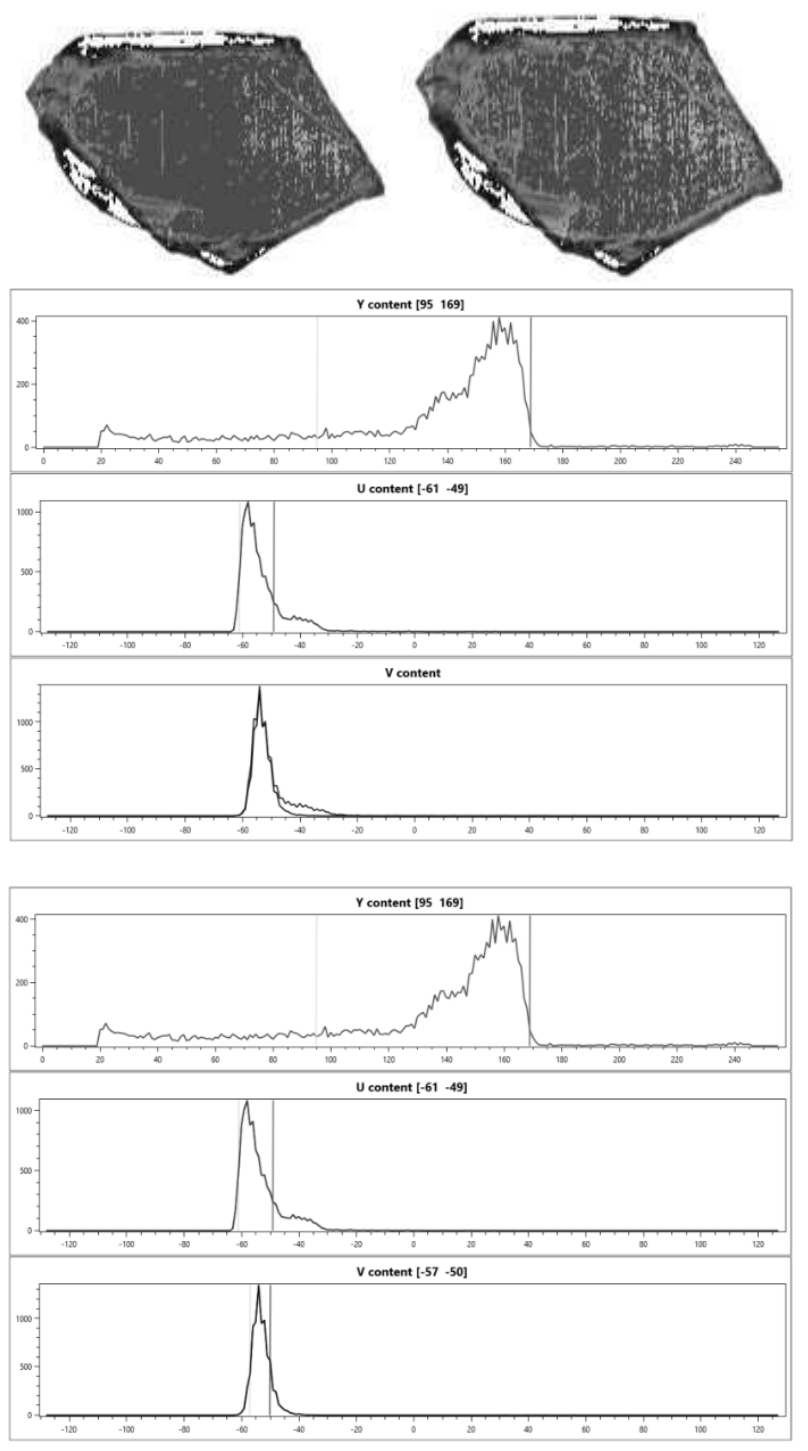

Fig. 11 Subsequent finding limits for $U$ and $V$ with visible smaller amount of pixels which satisfy (in red)

The found limits are hence (note that range for $\mathrm{Y}$ is 0 to 255 , range for $\mathrm{U}$ is -128 to 127 and range for $\mathrm{V}$ is -128 to 127$)$ :

Ylim00Do $=95$

Ylim00Up $=169$

Ulim00Do $=-61$

Ulim00Up $=-49$

Vlim00Do $=-57$

Vlim00Up $=-50$. 
When the limits are applied and with the assumption that the user chooses other important parameters obj_pres $=50 \%$ and col_pres $=50 \%$ the hit result is shown in Fig. 12. It is important to note that only a portion of the piece of glass is hit. It is also apparent from Fig. 13 that only one piece of glass is hit and the other pieces of glass are intact. This is the reason why there are up to 10 sets of such limits which are all tested. Hence we can save current limits and continue with different piece of glass of desired green color - Fig. 14.

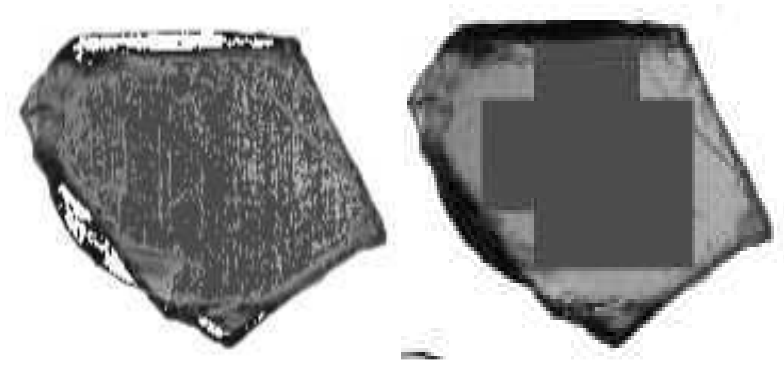

Fig. 12 Left - pixels which satisfy the $Y, U, V$ limits, right - hit results (valves which are enabled - red marks the places of the piece of glass where the air pressure is applied due to enabled valves)

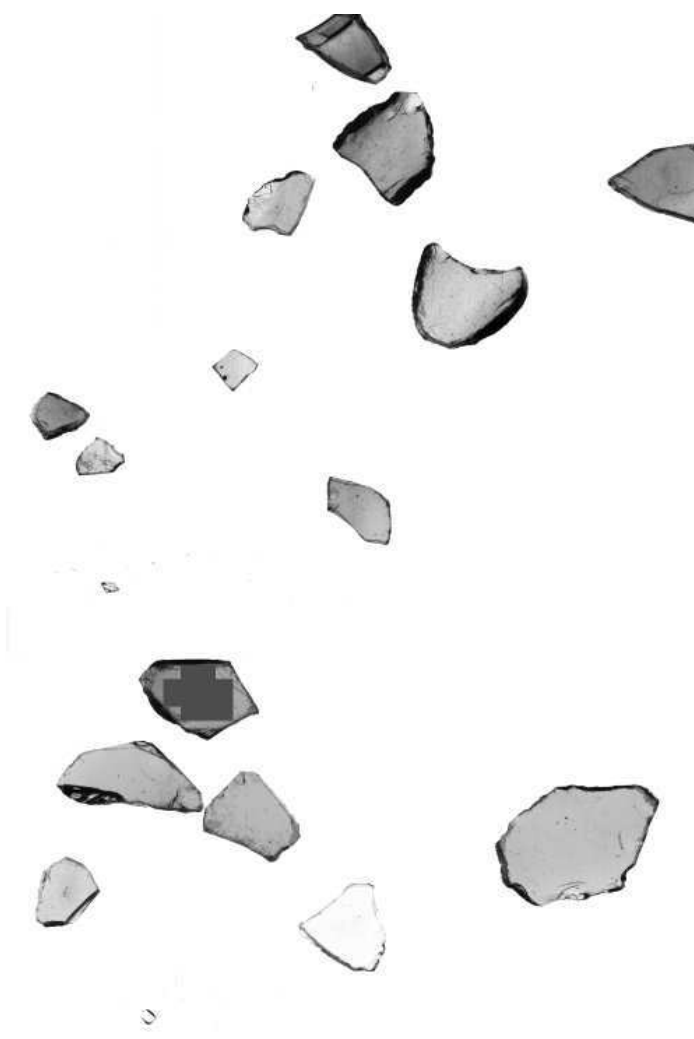

Fig. 13 Overall view of hits due to previously found conditions

Although we were finding optimal limits for only one piece of glass (Fig. 14 left), the pixels which satisfy the new conditions are also in other pieces (Fig. 14 center) and the resulting hits of pressured air is shown in Fig. 14 right. When so far 2 set of conditions are combined together the result is shown in Fig. 15 left. After adding 2 more conditions the result is in Fig. 15 right.

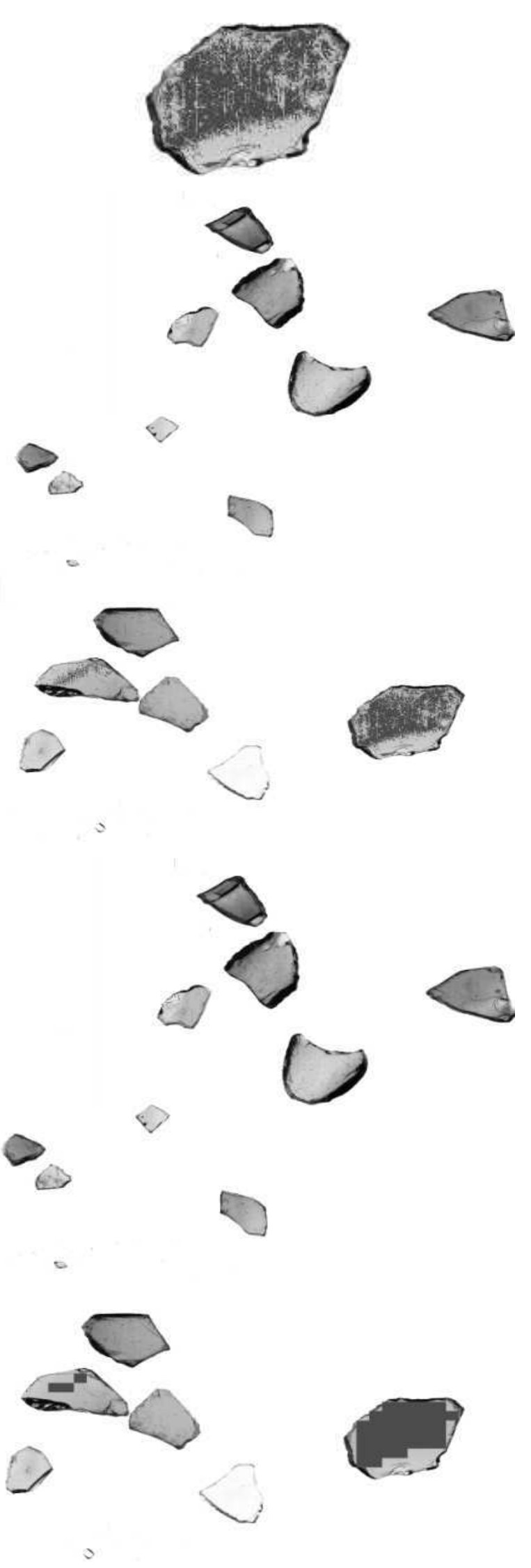

Fig. 14 Left - chosen piece of glass after black removal and application of $80 \% Y, U, V$ limits, center-overall pixel satisfaction for new set of limits, right-overall hit results 


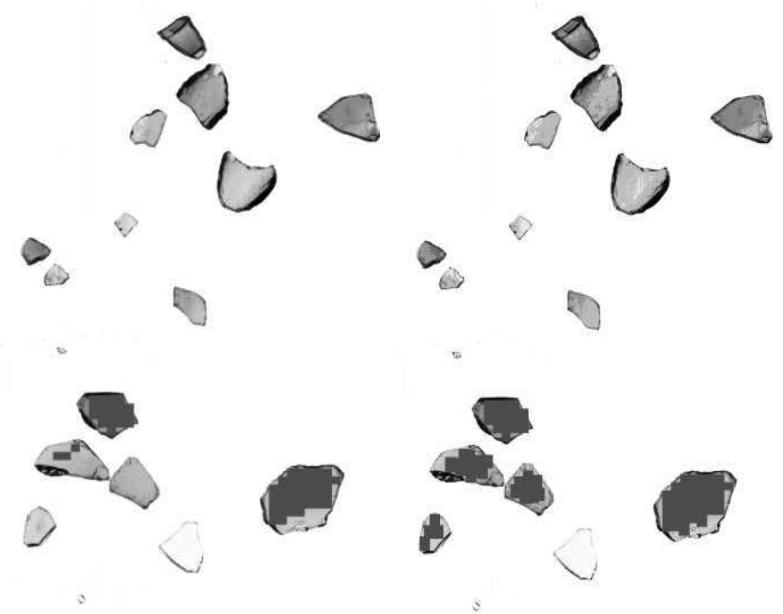

Fig. 15 Left - combined condition sets 1 and 2, rightcombined condition sets 1 through 4

The proposed method of finding the required limits depends only on the initial set of material for configuration. This is always available. The method convergence for finding the whole set of required $\mathrm{Y}, \mathrm{U}, \mathrm{V}$ limits is very fast - usually within 6 sets. The overall system is very robust. Let's suppose that during sorting the user finds out that some kind of green glass is not blown away. The only thing he has to do is to pick several pieces of such glass and add the configuration for that material to the current sets. User can also choose sorting several kinds of material at once (e.g. he can set the machine to blow away all green and brown glass). The system is also prepared and tested for reverse sorting - the demanded material is not blown out and the rest is blown out. The proposed FPGA structure enables such modifications very easily.

\section{Conclusion}

The proposed system is a complex sorting machine with all necessary parts: hardware, FPGA real-time communication, GUI for easy configuration and proposed and tested algorithms and techniques. Our goal was to enhance the availability of sorting universal sorting technology, which we believe is a way for solving part of current environment challenges. We have tested many times the efficiency of proposed device and algorithms. For CSP sorting the percentual efficiency is around 98\%. For color sorting the results are stated in Table 1.

Tab. 1 Efficiency of sorting

\begin{tabular}{|c|c|}
\hline Color component & Efficiency of sorting \\
\hline Green & $68 \%$ \\
\hline Brown & $74 \%$ \\
\hline Olive & $73 \%$ \\
\hline Transparent & $78 \%$ \\
\hline
\end{tabular}

A video of test when sorting is attached. It shows real environment during tests - conveyor belt, dirty cullet with a lot of CSP. The process of finding the Y, U, V limits is very important. So far we achieve this with manual setting of limits according to required portion of histograms. We would like to continue with full automatic configuration. The user would cast a fistful of glass to the sorting machine and mark the required pieces to be sorted out and the system would find alone all the necessary characteristics.

\section{Acknowledgement}

The result was obtained through the financial support of the Ministry of Education, Youth and Sports of the Czech Republic and the European Union (European Structural and Investment Funds - Operational Programme Research, Development and Education) in the frames of the project "Modular platform for autonomous chassis of specialized electric vehicles for freight and equipment transportation", Reg. No. CZ.02.1.01/0.0/0.0/16_025/0007293.

\section{References}

[1] MINKO, N.; BOLOTIN, V.; ZHERNOVAYA, N. (1999). Technological, energy, and environmental aspects of collecting and recycling of cullet (A Review). Glass and Ceramics, Vol. 56, N. 6, pp. 131133.

[2] Glass recycling hits $73 \%$ in the EU. [Internet]. Available from: http://feve.org/glass-recyclinghits-73-eu/ [Accessed: 16 July 2018]

[3] TESTA, M.; MALANDRINO, O.; SESSA, M.R.; SUPINO S. and SICA D. (2017). Long-Term Sustainability from the Perspective of Cullet Recycling in the Container Glass Industry: Evidence from Italy. Sustainability, Vol. 9, pp. 1752 1771.

[4] SILVA, R.V.; de BRITO, J.; LYE, C.Q.; DHIR, R.K. (2017). The role of glass waste in the production of ceramic-based products and other applications: A review. Journal of Cleaner Production, Vol. 167, N. 20, pp. 346-364.

[5] PYE, L.D.; STEVENS H.J.; La COURSE W.C. (eds) (1970). Introduction to Glass Science: Proceedings of a Tutorial Symposium. State University of New York.

[6] LIMBACHIYA, M. C.; LIMBACHIYA, J. R., editors (2004). Sustainable Waste Management and Recycling: Glass Waste - Vol. 1: Challenges and Opportunities; Kingston University London.

[7] STESSEL, R.I. (1996). Recycling and Resource Recovery Engineering: Principles of Waste Processing. Springer.

[8] DUBANOWITZ, A. J. (2000). Design of a Materials Recovery Facility (MRF) For Processing the Recyclable Materials of New York City's Municipal Solid Waste. M.S. thesis, Columbia University.

[9] BARNUM R. A. (2008). The Influence of Batch Segregation and Bulk Flow on Glass Quality. In: 66th Conference on Glass Problems (ed. Kriven, W.M). John Wiley \& Sons, pp: 91-103. 
[10] KIMMEL, K. S.; HAWK, N. A.; KELLER, M.A. WHITMORE, F. (2002). Patent US6464082. Cullet sorting using density variations.

[11] KIMMEL, K. S.; HAWK, N. A. (2000). Patent US6112903. Cullet sorting by differential thermal characteristics.

[12] STIRLING, H. (1977). The recovery of waste glass cullet for recycling purposes by means of electro-optical sorters. Conservation \& Recycling, Vol.1, N. 2, pp. 209-219.

[13] AFSARI, F.; AFSARI, B.; FLYNN, P. C.; KOPELIOFF, D.; SHOOK, F. S.; VENDRELL, L. P. (2002). Patent US8436268B1. Method of and apparatus for type and color sorting of cullet.

[14] STELTE, N. (1994). Patent US5333739. Method and apparatus for sorting bulk material.

[15] REICHERT, A.; HOBERG, H. (1987). Photometric Sorting of cullet. In: Environmental Technology: Proceedings of the Second European Conference on Environmental Technology (eds. de Waal, K.J.A.; van den Brink, K.J.A.), Springer, pp. $457-465$.

[16] BONIFAZI, G.; SERANTI, S. (2006). Imaging spectroscopy based strategies for ceramic glass contaminants removal in glass recycling. Waste Management. Vol. 26, N.6, pp. 627-639.

[17] Redwave CX / CXF. [Internet]. Available from: http://www.redwave.com/en/products/redwavecx-cxf/ [Accessed: 16 July 2018]

[18] BONIFAZI, G. (2004). Classical Imaging and Digital Imaging Spectrophotometric Techniques in Cullets (Glass Fragments) Sorting. In: Intelligent Robots and Computer Vision XXII: Algorithms, Techniques, and Active Vision (eds. Casasent, D. P.; Hall E. L.; Röning, J.), SPIE, pp. 264-277.

[19] CRAPARO, J. C.; WEISBERG, A.; de SARO, R. (2008). Measurements of Batch and Cullet Using
Laser Induced Breakdown Spectroscopy. In: 66th Conference on Glass Problems (ed. Kriven, W.M.), John Wiley \& Sons, pp. 105-118.

[20] SUMINA, K.; AKAI, T., YAMASHITA, M; YAZAWA T. (2004). Patent JP2004219125. Method for sorting glass cullet and sorting apparatus therefor.

[21] HUBER, R.; PANSINGERM, C. (2007). Patent application AU2006203360A1. A method for detecting and sorting glass.

[22] HUBER, R. (2013). Waste glass sorting based on UV absorption and fluorescence. In: 16th SpectroNet Collaboration Forum, Karlsruhe.

[23] Redwave XRF-G. [Internet]. Available from: http://www.redwave.com/en/recycling/glass/sensor-based/redwave-xrf-g/ [Accessed: 16 July 2018]

[24] GUO, C.S.; YU, Y.N.; HONG, Z. (2010). Optical sorting using an array of optical vortices with fractional topological charge. Optics Communications, Vol. 283, N. 9, pp. 1889-1893.

[25] MAJERNÍK, J.; KMEC, J.; KARKOVA, M.; PODAŘIL, M. (2017). Possibilities for change of thermoplastic tensile properties using admixture of recyclable material. Manufacturing Technology, Vol. 17, N. 5, pp. 778-782.

[26] PETRŮ, M.; MARTINEC, T.; MLÝNEK, J. (2016). Numerical model description of fibres winding process for new technology of winding fibres on the frames. Manufacturing Technology, Vol. 16, N. 4, pp. 778-785.

[27] PETRŮ, M.; MLÝNEK, J.; MARTINEC, T. (2018). Numerical modelling for optimization of fibres winding process of manufacturing technology for the non-circular aerospaces frames. $\mathrm{Ma}$ nufacturing Technology, Vol. 18, N. 1, pp. 90-98. 